\title{
LA TRAMPA DE LIQUIDEZ, HISTORIA Y TENDENCIAS DE INVESTIGACIÓN: UN ANÁLISIS BIBLIOMÉTRICO
}

\author{
Eva Ugarte, Josefina León y Gilberto Parra ${ }^{1}$
}

Fecha de recepción: 25 de noviembre de 2016. Fecha de aceptación: 20 de marzo de 2017.

\begin{abstract}
RESUMEN
En la trampa de liquidez, la política monetaria expansiva convencional es ineficaz para alentar la actividad económica. El modelo keynesiano ortodoxo plantea una situación de este tipo como un caso extremo, el cual fue utilizado para justificar la importancia de la política fiscal. El objetivo de este artículo consiste en realizar un análisis cuantitativo (mediante técnicas bibliométricas) y cualitativo para explorar la literatura reciente sobre la trampa de liquidez. La evolución del número de publicaciones en el periodo 19732015 devela los eventos económicos (depresión japonesa de los años noventa y crisis financiera mundial de 2007-2008) que renovaron el interés académico en el estudio de este tema. Estos resultados ofrecen una visión esquemática muy acertada sobre la actual agenda de investigación.
\end{abstract}

Palabras clave: trampa de liquidez, tasa de interés nominal, política monetaria, política fiscal, bibliometría, teoría keynesiana.

Clasificación JEL: C88, E43, E52, E63.

\section{The Liquidity Trap, History, and Research Trends: A Bibliometric Analysis}

\begin{abstract}
In a liquidity trap, the conventional expansionary monetary policy is not an effective way to stimulate economic activity. The orthodox Keynesian model poses a situation of this sort as an extreme case, which has been used to justify the importance of fiscal policy. This paper aims to conduct a qualitative and quantitative analysis (using bibliometric techniques) to explore the recent literature about the liquidity trap. The way in which the number of publications in the period 1973-2015 evolved points to the economic events (Japanese depression in the nineteen-nineties and the 2007-2008 global financial crisis) that revived academic interest in studying this topic. We believe that these results offer a very accurate schematic overview of the current research agenda.
\end{abstract}

Key Words: Liquidity trap, nominal interest rate, monetary policy, fiscal policy, bibliometrics, Keynesian theory.

1 Universidad Autónoma Metropolitana, México. Correos electrónicos: ugarteva@gmail.com, leon2josefita@hotmail.com y pahug@outlook.com, respectivamente. 


\section{TRAPPE À LIQUIDITÉ, HISTOIRE ET TENDANCES DE LA RECHERCHE : UNE APPROCHE BIBLIOMÉTRIQUE \\ Résumé}

Dans la trappe à liquidité la politique monétaire expansive conventionnelle est inefficace pour relancer l'activité économique. Le modèle keynésien orthodoxe considère cette dernière situation comme un cas extrême, utilisé pour justifier l'importance de la politique budgétaire. Le but de cet article consiste à réaliser une analyse quantitative (par des techniques bibliométriques) et qualitative pour examiner la littérature économique récente par rapport à la trappe à liquidité. L'évolution du nombre de publications dans la période 1973-2015 révèle les évènements économiques (la dépression japonaise des années quatre-vingt-dix et la crise financière mondiale de 2007-2008) qui ont relancé l'intérêt académique pour l'étude de ce sujet. On considère que les résultats apportent un point de vue schématique très sensé sur l'agenda actuel de recherche.

Mots clés: trappe à liquidité ; taux d'intérêt nominal ; politique monétaire ; politique budgétaire ; bibliométrie, théorie keynésien.

\section{ARMADILHA DA LIQUIDEZ, HISTÓRIA E TENDÊNCIAS DE PESQUISA: UMA ANÁ- LISE BIBLIOMÉTRICA \\ Resumo}

$\mathrm{Na}$ armadilha da liquidez, a política monetária convencional é ineficaz para estimular a atividade econômica. $\mathrm{O}$ modelo keynesiano ortodoxo apresenta uma situação deste tipo como um caso extremo, que foi usado para justificar a importância da política fiscal. O objetivo deste trabalho é apresentar uma análise quantitativa (usando técnicas bibliométricas) e qualitativa para explorar a literatura recente sobre a armadilha da liquidez. A evoluçáo do número de publicaçóes no período 1973-2015 revela os eventos econômicos (depressão japonesa dos noventa e crise financeira global de 2007-2008) que renovaram o interesse acadêmico em estudar este tema. Acreditamos que estes resultados fornecem uma visão esquemática muito precisa da agenda de pesquisa atual.

Palavras-chave: armadilha da liquidez; taxa nominal de juros; política monetária; política fiscal; bibliometria, teoria keynesiana.

流动性陷阱历史及其研究动向的计量分析

伊娃・格特 何塞菲娜・莱昂 基尔伯特・帕拉

摘要

在流动性陷吽中，传统的货币扩张政策对刺激经济活动是无效的。正统的 凯恩斯模型提出了此种情况下的一个极端例子来证明财政政策的重要性。 本文旨在通过文献计量技术进行定量和定性分析来探索有关流动性陷阱的 最新研究文献。1973-2015年间所发表文献数量的变化揭示了诸如90年 代的日本经济衰退及2007-2008年国际金融危机等经济事件，从而激起了 关于这一议题的新的学术兴趣。我们认为这些研究结果为当今的研究议题 提供了一个非常精准的概述性观点。

关键词：流动性陷阱、利率、货币政策、财政政策、文献计量学、凯恩斯 理论 


\section{INTRODUCCIÓN}

La prolongada etapa de estancamiento económico que ha sufrido Japón desde la década de los noventa, así como el estallido de la crisis financiera mundial de 2007-2008 con epicentro en Estados Unidos, despertaron el interés de los economistas por analizar un viejo concepto: la trampa de liquidez. ${ }^{2} \mathrm{Se}$ trata de un término cuyo origen se remonta a las discusiones teóricas surgidas a raíz de la publicación de la famosa obra de J. M. Keynes (1936), en el contexto de la Gran Depresión. ${ }^{3}$

La depresión mundial que marcó la década de los treinta es considerada la peor catástrofe económica del siglo xx. El derrumbe de la Bolsa de Valores de Nueva York, en octubre de 1929, fue el anuncio de una etapa de severa contracción en la producción agregada y aumento del desempleo en Estados Unidos, principalmente en el periodo 1931-1933. La Gran Depresión estadounidense se caracterizó por la presencia de tasas de interés nominal de corto plazo muy bajas. Por ejemplo, la tasa de interés de los títulos del Tesoro a tres meses en el mercado secundario (3-month Treasury bill: secondary market rate) alcanzó un nivel de $0.15 \%$ en abril de 1934, llegó a $0.56 \%$ en abril de 1937 y descendió a $0.05 \%$ en junio de $1938 .^{4}$

En la jerga económica se dice que hay una trampa de liquidez cuando la política monetaria expansiva convencional pierde su capacidad para estimular la demanda agregada y/o para incidir sobre el nivel de precios. Una situación de esta naturaleza se presenta cuando la tasa de interés nominal ha alcanzado su límite inferior o se encuentra cerca de él. Dicho límite puede concebirse como un valor pequeño pero positivo o puede ser igual a cero. ${ }^{5}$ Como se verá

Aunque el término como tal fue acuñado por Robertson (1940), un fuerte crítico de la teoría de determinación de la tasa de interés de Keynes, el significado que dicho autor le atribuyó dista del que actualmente conocemos. En realidad, el concepto trampa de liquidez se asocia con la interpretación de la teoría de preferencia por liquidez de Keynes plasmada por Hicks (1937) en su artículo "Mr. Keynes and the 'Classics' a Suggested Interpretation", donde la llamó "economía de la depresión".

Cabe señalar que en la Teoría General de la Ocupación, el Interés y el Dinero, Keynes no se refiere explícitamente a la Gran Depresión, sin embargo, se sabe que el propósito de dicha obra fue dar una respuesta teórica a las dificultades que enfrentaron las principales economías capitalistas a partir del "crash de 1929".

https://research.stlouisfed.org/fred2/series/TB3MS\#

Por ejemplo, Hicks (1937), hacía referencia a una tasa de interés pequeña pero positiva, mientras que la literatura actual apunta al límite inferior cero de la tasa de interés nominal. Boianovsky (2003) atribuye esta diferencia a la tasa de interés que se considera en cada modelo; así, mientras que el modelo IS/LL de Hicks enfatiza la tasa de interés nominal de largo plazo, el modelo de Krugman (1998) utiliza la tasa de interés nominal de corto plazo (de un periodo) como la tasa relevante. 
más adelante, además de la presencia de tasas de interés nominales muy bajas o nulas, en la literatura reciente, la trampa de liquidez se asocia también con tasas de inflación muy bajas o incluso negativas, ${ }^{6}$ de manera que el Banco Central ya no puede ajustar su política ante shocks deflacionarios mediante recortes en la tasa de interés nominal.

"Las lecciones de la historia del pensamiento económico nos enseñan que una de las principales fuerzas que guían la evolución de nuevas ideas es la marcha de los acontecimientos" (Snowdon \& Vane, 2005, p. 9). En efecto, el concepto de trampa de liquidez que hemos señalado es producto de las contribuciones de notables economistas y es un reflejo de la evolución del pensamiento económico, desde la macroeconomía personificada en sus inicios por el modelo IS/LM (que incluye las contribuciones de J. R. Hicks, F. Modigliani, D. Patinkin, J. Tobin, entre otros), hasta los modelos "neokeynesianos" (Clarida et al., 1999) de equilibrio general dinámico estocástico de nuestros días. $^{7}$

En el modelo IS/LM la trampa de liquidez desempeña un papel importante porque pone de relieve a la política fiscal como instrumento para alentar la actividad económica, ya que en este escenario no existe el efecto crowding out. Esto no es un asunto menor, si tomamos en cuenta que uno de los principales postulados que separan a los economistas keynesianos de los neoclásicos es la defensa que hacen los primeros de la necesidad de intervención gubernamental, a través de la política fiscal, para influir en la marcha de la actividad económica.

Sin embargo, los eventos económicos que se presentaron a finales de la década de los sesenta y principios de los setenta (la estanflación y el derrumbe del Sistema Monetario de Bretton Woods) dieron lugar al cuestionamiento de las ideas keynesianas. En consecuencia, desde finales de la década de los sesenta y durante toda la década de los setenta, dos corrientes de pensamiento económico dominaron la escena: el Monetarismo y la Nueva Escuela Clásica, ambas corrientes por igual restaron importancia al concepto de trampa de liquidez aunque sostenían diferentes razones. De acuerdo con los monetaristas la política monetaria tiene varios canales de transmisión además de la tasa de

6

Aunque la asociación entre el problema de la deflación y la trampa de liquidez la encontramos, principalmente, en investigaciones recientes sobre política monetaria, también Hicks (1939) señaló esta relación.

Este tipo de modelos también es conocido en la literatura económica como neowicksellianos (Woodford, 2003) o de la Nueva Síntesis Neoclásica (Goodfriend \& King, 1997). El esquema analítico está formado, básicamente, por una ecuación IS intertemporal, una regla de política monetaria y una ecuación de oferta agregada representada por la curva de Phillips neokeynesiana. 
interés. Por tanto, aun cuando este canal estuviera limitado (es decir, que los impulsos monetarios no se pudieran transmitir a través de la tasa de interés porque ésta se encuentra ya en un nivel muy bajo), es poco probable que también se elimine el efecto que la política monetaria tiene sobre el precio de los diferentes activos que existen en la economía (activos reales y financieros). Así, los monetaristas rechazan la posibilidad de que a niveles bajos de tasa de interés la política monetaria pierda su efectividad en el corto plazo.

Es desde la perspectiva de los nuevos clásicos, que la trampa de liquidez es un problema que carece de sentido. Según esta corriente teórica, todas las fluctuaciones en la actividad económica (incluso las caídas) son equilibrios competitivos; es decir, se consideran situaciones óptimas por lo que no constituyen un problema a resolver. Además, debido a que los mercados siempre se vacían (incluyendo el mercado de trabajo) todo el desempleo que existe es voluntario. En este escenario, la economía nunca puede estar restringida por un déficit de demanda efectiva como lo argumentaba Keynes (1936). La política fiscal es innecesaria y se arguye en favor de la mínima intervención del gobierno debido a la confianza que se tiene en el mecanismo de autoajuste de los mercados.

En cuanto al escenario económico, durante estas décadas las tasas de interés en los países desarrollados se mantuvieron en niveles elevados. Por ejemplo, desde julio de 1963 en adelante, la tasa de interés de los títulos del Tesoro estadounidense a tres meses nunca fue inferior a 3\%. En enero de 1970 fue de $7.87 \%$, en agosto de 1973 llegó a 8.67\% y en enero de 1980 alcanzó 12\%. Todo lo anterior ofrece elementos para entender por qué desde finales de los ańos sesenta casi toda referencia a la trampa de liquidez fue relegada de la literatura económica.

No obstante, diversos acontecimientos (entre ellos la reforma al sector financiero japonés y la apreciación del tipo de cambio nominal del yen con respecto al dólar estadounidense) afectaron el desempeño económico de Japón en los ańos noventa, dando lugar a una etapa de estancamiento que persiste hasta la fecha. De acuerdo con cifras del Banco de Japón (BJ), en el periodo 1992-2003 la tasa de crecimiento anual promedio del producto interno bruto (рів) japonés fue de $0.9 \%$, lo que condujo a la implementación de una política monetaria laxa. Además, una característica particular de la crisis japonesa de esos años fue la deflación. Estos acontecimientos revivieron el interés académico por estudiar el problema de la trampa de liquidez (el ejemplo más notable es el de Krugman, 1998).

Por otro lado, el cambio de la política económica mundial hacia la desregulación de los mercados financieros ha propiciado profundas transforma- 
ciones. La crisis de 2007-2008 es en gran parte resultado de este proceso. El origen de la crisis se encuentra a principios de la década de 2000 con el auge especulativo en el sector de bienes raíces, propiciado en parte por una excesiva liquidez monetaria (debido a la política de bajas tasas de interés) y una mala supervisión en el otorgamiento de créditos (hipotecas subprime). ${ }^{8} \mathrm{El}$ incremento en las tasas de interés que implementó la Reserva Federal (FED) en la segunda mitad de 2004 y, que continuó durante 2005 y 2006, ${ }^{9}$ pronto se reflejó en el aumento en la tasa de morosidad de los préstamos hipotecarios.

El estallido de la burbuja inmobiliaria en Estados Unidos comenzó en julio de 2007 cuando se declaró la quiebra de los fondos hipotecarios del banco de inversión Bear Stearns, lo que generó gran incertidumbre entre los inversionistas. Para septiembre de 2008 los mercados financieros se derrumbaron completamente. Los estragos de la crisis se resintieron rápidamente en la economía real: en 2009 la tasa de desempleo alcanzó $9.6 \%$ y la tasa de crecimiento del PIB real fue de $-3.0 \%$. Con la recesión en puerta, a finales de 2008 la FED se vio obligada a reducir la tasa de fondos federales y desde entonces la ha mantenido en niveles inusualmente bajos, lo que ha dado un nuevo impulso a la investigación académica de la trampa de liquidez.

Dada la reciente notoriedad que ha cobrado el concepto, este artículo analiza la trampa de liquidez utilizando los métodos de la bibliometría. Actualmente existen otros trabajos que se han valido de esta metodología para identificar las principales tendencias y estudiar la estructura de algunos campos de investigación económica (por ejemplo, Prado et al., 2016; Hsu \& Chiang, 2015; Chun-Hao \& Jian-Min, 2012).

El artículo se estructuró de la siguiente manera: en la sección 2 se describen las fuentes de información y la metodología empleada para su procesamiento mediante herramientas de software especializado; la sección 3 contiene un análisis de la evolución de las ideas relacionadas con el concepto de trampa de liquidez durante el periodo establecido (1973-2015) y de los sucesos económicos con los que se asocian; un mapa de las principales palabras clave extraídas de los documentos de la base de datos que se complementa con una

9

Carlos Obregón (2011) presenta un análisis detallado de la crisis financiera mundial y las perspectivas de México y América Latina.

En el mes de diciembre de 2000, la tasa efectiva de fondos federales era de $6.4 \%$ y en el mismo mes del siguiente año ya se había reducido hasta $1.82 \%$. Esta tasa alcanzó su nivel más bajo en diciembre de 2003 al ubicarse en $0.98 \%$ y, aunque en los meses siguientes tuvo pequeños aumentos, se mantuvo por debajo del 2\%. Sin embargo, en diciembre de 2004 subió a 2.16\%. A partir de esa fecha, los incrementos continuaron hasta llegar a 5.25\% en agosto de 2006 (datos del Banco de la Reserva Federal de St. Louis https://research.stlouisfed.org/fred2/series/FEDFUNDS\#). 
explicación de la relación que éstas guardan con la trampa de liquidez. Finalmente, el análisis llega a su conclusión, en la sección 4, e identifica cuáles son las implicaciones centrales en torno al papel de las políticas monetaria y fiscal en una trampa de liquidez en los modelos neokeynesianos.

\section{FUENTES Y METODOLOGÍA}

Los artículos utilizados en esta investigación se obtuvieron de la base de datos Scopus de Elsevier, misma que contiene una gran cantidad de resúmenes y referencias bibliográficas de la literatura científica revisada por pares. Scopus agrupa más de 18 mil títulos de 5 mil editoriales internacionales. ${ }^{10}$ Los documentos científicos pueden descargarse y procesarse en herramientas de software especializado para analizar la estructura de un campo científico. ${ }^{11}$

Para identificar los artículos acerca de la trampa de liquidez se utilizaron los siguientes términos: liquidity trap, zero lower bound o zero interest rate. Se seleccionaron los artículos que contuvieran al menos uno de los términos en el título, resumen o palabras clave. Posteriormente, se eligieron únicamente los artículos y las revisiones que pertenecieran al área temática de Economics, Econometrics and Finance. El periodo de análisis se estableció desde 1973, año con el primer registro, hasta 2015. Al aplicar esos filtros se obtuvieron 383 documentos.

Después de identificar los documentos se descargaron y analizaron con el software VOSviewer (Van Eck \& Waltman, 2010 y 2011). Se seleccionaron solamente las palabras clave anotadas por los autores y que aparecieran por lo menos en cinco publicaciones. De los 383 artículos, solamente 265 contaban con palabras clave anotadas por los autores. Fue a partir de esa información que se elaboró un mapa de dos dimensiones (véase figura 1$){ }^{12}$

El VOSviewer permite calcular, para cada palabra clave, una puntuación basada en el año en que se publicaron los artículos donde las palabras aparecen. Para calcular ese valor se suman los años y se dividen entre el número de artículos. ${ }^{13}$ Para efectos del análisis, los términos que obtienen una puntua-

10

11

12

13

http://www.americalatina.elsevier.com/corporate/es/scopus.php

Entre esas herramientas encontramos a Gephi, Pajek, VOSviewer o CiteSpace (Van Eck \& Waltman, 2014).

La distribución de las palabras en el mapa se determinó utilizando la técnica visualization of similarities (vos); la cual, según sus autores, produce mejores representaciones que el escalamiento multidimensional (Van Eck et al., 2010).

Por ejemplo, si una palabra clave aparece en un artículo publicado en el 2015 y también aparece en dos artículos publicados en 2013, entonces el valor para esa palabra será de 2013.66. 
ción baja son los que aparecen en artículos más antiguos. Por el contrario, los que tienen una puntuación elevada pertenecen a los temas que guían la actividad más reciente en el área de investigación.

\section{RESULTADOS}

El interés por estudiar la trampa de liquidez exhibe una tendencia creciente en las últimas décadas. La gráfica 1 muestra que hasta antes del año 2000 las publicaciones relacionadas con este tema fueron esporádicas, pero a partir de esa fecha se observó un primer repunte en el número de publicaciones por año. Lo anterior se explica por el estallido de la burbuja financiera en Japón en 1991 y el inicio de una prolongada etapa de estancamiento económico que persiste hasta la actualidad en dicho país. Como señalamos antes, el débil crecimiento del PIв japonés durante la década de los noventa obligó al вј а relajar su política monetaria. En julio de 1995 la tasa de interés nominal de corto plazo (medida por el promedio mensual de la Uncollateralized Overnight Call Rate, UOCR) cayó a $0.95 \%$, y en marzo de 1999 llegó a $0.04 \% .{ }^{14}$ Con la tasa de interés en su nivel mínimo, el Banco Central se vio obligado a recurrir a una política de relajamiento cuantitativo entre los ańos de 2001 y 2006 con el fin de incrementar los precios y estimular la demanda agregada.

No obstante, la tasa de inflación fue apenas positiva en los años 2006, 2007 y $2008(0.243,0.061$ y $1.378 \%$, respectivamente) y volvió a valores negativos entre los años de 2009 y $2012 .{ }^{15}$

Posteriormente, hubo un incremento sostenido en el número de publicaciones entre los años 2002 y 2005 que se asocia, principalmente, a la situación económica de Estados Unidos y a la reducción al 1\% de la tasa de interés de referencia de la FED en junio de 2003. Sin embargo, el crecimiento más notable se presenta a partir del ańo 2009 , lo que se atribuye a los efectos de la crisis financiera global de 2007-2008.

Es pertinente destacar que el escaso número de publicaciones sobre la trampa de liquidez en las décadas de los setenta, ochenta y noventa es consistente con los dos factores que señalamos en la introducción: el surgimiento de corrientes del pensamiento económico adversarias de la visión keynesiana

Datos del Banco de Japón. La serie de tiempo con estos datos se encuentra con el código ST'STRACLUCON en la página <http://www.stat-search.boj.or.jp/ssi/cgi-bin/famecgi2? cgi $=\$$ nme_s050_en>

Cifras del World Economic Outlook Database del Fondo Monetario Internacional (FMI). 
Gráfica 1. Evolución del número de publicaciones sobre la trampa de liquidez

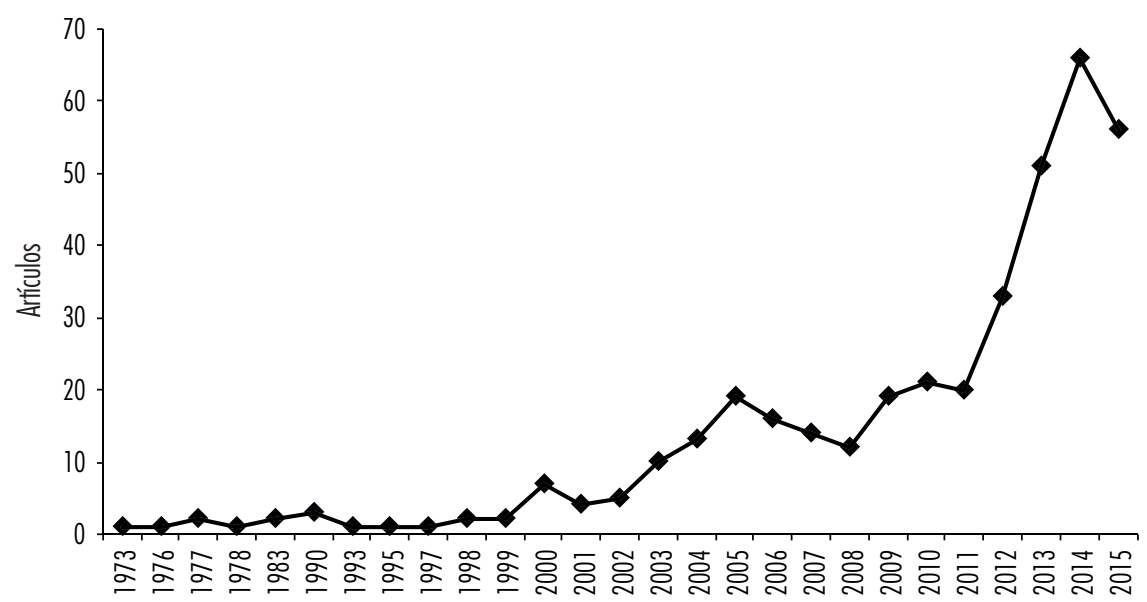

Fuente: elaboración propia con datos de Scopus.

tradicional y el escenario económico caracterizado por elevadas tasas de interés en los países desarrollados. Estos elementos explican por qué desde finales de los años sesenta hasta los albores del nuevo milenio, la trampa de liquidez fue irrelevante en la literatura económica.

\subsection{Frecuencia y año promedio de aparición de las palabras clave}

Las puntuaciones del cuadro 1 proporcionan información que contribuye a explicar la gráfica 1 y complementan nuestro análisis de palabras clave del siguiente apartado. Respecto a los temas actuales vemos que el multiplicador fiscal (fiscal multiplier) se ha colocado en primer lugar, aunque aún aparece en pocos artículos. También observamos que la mayoría de los términos tiene un año promedio superior al del año de 2008, lo que nos indica que están relacionados, principalmente, con la crisis financiera (financial crisis es un término que también aparece en el mapa de la figura 1) de los últimos años y la política monetaria implementada por el Banco Central estadounidense (Federal Reserve).

Es conocido que a raíz del derrumbe de los mercados financieros en septiembre de 2008 y con la recesión en puerta, la FED puso en marcha una serie de medidas para incentivar la recuperación económica y la generación de empleo. Una de ellas fue la modificación de la tasa de fondos federales que 
Eva Ugarte, Josefina León y Gilberto Parra

Cuadro 1. Datos relevantes sobre los términos clave obtenidos de vosviewer

\begin{tabular}{|c|c|c|}
\hline Término & Frecuencia & Promedio \\
\hline Fiscal multiplier & 5 & 2014.4 \\
\hline New Keynesian Model & 5 & 2014.2 \\
\hline Unconventional monetary policy & 9 & 2013.6 \\
\hline Zero lower bound & 65 & 2013.0 \\
\hline Federal Reserve & 5 & 2012.8 \\
\hline Quantitative easing & 24 & 2012.5 \\
\hline Fiscal policy & 23 & 2012.0 \\
\hline Zero interest rate & 6 & 2012.0 \\
\hline Monetary policy & 71 & 2011.8 \\
\hline Interest rate & 6 & 2011.5 \\
\hline Financial crisis & 7 & 2011.4 \\
\hline Inflation targeting & 9 & 2011.3 \\
\hline Taylor rule & 9 & 2011.3 \\
\hline Interest rates & 8 & 2010.8 \\
\hline Zero interest rate policy & 9 & 2010.7 \\
\hline Inflation & 10 & 2010.2 \\
\hline Optimal monetary policy & 6 & 2009.7 \\
\hline Money & 7 & 2009.6 \\
\hline Liquidity trap & 66 & 2008.8 \\
\hline China & 7 & 2007.6 \\
\hline Money demand & 6 & 2007.2 \\
\hline Japan & 10 & 2007.1 \\
\hline Zero bound & 12 & 2007.1 \\
\hline Deflation & 21 & 2006.8 \\
\hline Monetary policy rules & 5 & 2006.2 \\
\hline
\end{tabular}

Nota: La frecuencia es el número de artículos en los cuales aparece cada término. Promedio es el año promedio de los artículos donde aparece el término.

Fuente: elaboración propia. 
en diciembre de 2008 se redujo a niveles históricamente bajos de entre 0 y $0.25 \%$ y se mantuvo así en los años posteriores. Fue hasta el 17 de diciembre de 2015 cuando aumentó ligeramente en un cuarto de punto para ubicarse en un intervalo de 0.25 a $0.5 \%$. La ineficacia de la política monetaria en este escenario de tasas de interés tan bajas, obligó a la FED a recurrir a medidas de expansión monetaria no convencionales como su programa de relajamiento cuantitativo (quantitative easing) también conocido como Large Scale Asset Purchases (LSAPS, por sus siglas en inglés) ${ }^{16}$ que inició en noviembre de 2008. Hasta la fecha, Estados Unidos ha efectuado tres rondas de relajamiento cuantitativo y la tercera fue suspendida a partir de octubre de $2014 .^{17}$

En la parte baja del cuadro aparece Japón (Japan), lo cual también es consistente con los problemas económicos que ese país enfrenta desde la década de los noventa. Además, es importante tener en cuenta que a pesar de que la literatura actual está más enfocada en el caso estadounidense, Japón sigue en la escena pues aunque la llamada Abe-nomics ha tenido algún efecto, la economía de dicho país no ha podido retomar una senda de crecimiento económico.

Entre las medidas de política monetaria que destacan con la llegada de Abe Shinzo como primer ministro japonés en diciembre de 2012, están la adopción de un objetivo de inflación de $2 \%$ y el inicio del tercer programa de relajamiento cuantitativo en abril de 2013. Es pertinente señalar que el вј fue el primero en el mundo en implementar una política de relajamiento cuantitativo. Esto sucedió en marzo de 2001, después de que en el mismo mes del año previo la tasa de interés de préstamos a corto plazo en el mercado interbancario japonés ${ }^{18}$ llegara a cero. Asimismo, la deflación (deflation) es una de las

Aunque generalmente se hace alusión a este programa de expansión monetaria como relajamiento cuantitativo, de acuerdo con Bernanke (2009), se trató más bien de un relajamiento del crédito (credit easing), ya que el objetivo de dicho programa no era simplemente incrementar la base monetaria (como ocurrió en Japón entre 2001 y 2006), sino que estaba enfocado a mejorar el funcionamiento del mercado de bonos de largo plazo y disminuir las tasas de interés de largo plazo. En este sentido, se dice que alrededor del $80 \%$ de los activos comprados por la FED en su primer programa de "relajamiento cuantitativo" estuvieron vinculados directamente al mercado de la vivienda. Pero no sólo la FED emprendió medidas de expansión monetaria no convencionales, también el BJ, el Banco Central Europeo y el Banco de Inglaterra implementaron políticas de relajamiento cuantitativo entre finales de 2008 y principios de 2009 (Fawley \& Neely, 2013).

La tasa de interés que el вј utilizaba como su instrumento operativo de política monetaria era la Uncollateralized Overnight Call Rate (UOCR), pero con el inicio del primer programa de relajamiento cuantitativo $(\mathrm{QE})$ dicho instrumento fue sustituido por el nivel absoluto de los saldos en cuenta corriente que mantienen los bancos comerciales en el Banco Central (conformados por las reservas bancarias más depósitos de las instituciones financieras no bancarias) (Ashworth, 2013). Sin embargo, en marzo de 2006 cuando concluyó el QE se reinstaló la UOCR como el principal instrumento de política monetaria. 
palabras clave que cobraron gran relevancia en el contexto de la recesión japonesa. La tasa de inflación anual en Japón en 1995 fue de $-0.125 \%$ y las cifras negativas se observaron también entre 1999 y $2005(-0.328,-0.651,-0.804$, $-0.9,-0.25,-0.01$ y $-0.274 \%$, respectivamente) ${ }^{19}$ Un análisis detallado de las causas y consecuencias de este aspecto se encuentra en Svensson (2003).

En este contexto, vale la pena observar que la política monetaria que se ha aplicado en Estados Unidos y otros países desarrollados a partir de la crisis financiera mundial y que ha continuado hasta la actualidad, muestra limitaciones como vía de impulso al crecimiento económico y la generación de empleo. Lo anterior, en un contexto en el que sigue prevaleciendo la incertidumbre en los mercados financieros internacionales y donde la trampa de liquidez se asocia con problemas de deflación en los mercados de bienes y servicios. En contraste, se observa un ascenso en los precios de los activos financieros (como acciones y bonos) en los mercados especulativos, aspecto considerado por los monetaristas cuando señalan que la política monetaria tiene efectos en los precios de los diferentes activos, tanto reales como financieros.

Asimismo, en la actualidad existe una falta de coordinación en la política monetaria a nivel internacional. Es decir, mientras Japón continúa con su política monetaria extremadamente laxa y una situación similar se observa en los países de la Eurozona e Inglaterra, Estados Unidos eleva ligeramente la tasa de interés y suspende el programa de flexibilización cuantitativa.

Otros temas recientes que destacan en el cuadro por su frecuencia de aparición son la política monetaria y fiscal, que analizamos con más detalle en el siguiente apartado con base en los artículos más citados.

\subsection{Red de palabras}

El mapa de palabras clave de la figura 1 pone de relieve los conceptos importantes asociados con la discusión académica actual sobre la trampa de liquidez, así como la fuerza de la relación que existe entre ellos. El tamaño de las palabras en el mapa está en función de la ocurrencia del término y el grosor de los enlaces está determinado por el número de veces que los pares de palabras aparecen juntas. A continuación presentamos un análisis de los términos que aparecen en el mapa, el cual se elaboró con base en los 12 artículos más citados en Scopus y que se listan en el Anexo de este documento. Cabe señalar que el 
Figura 1. Mapa de palabras clave

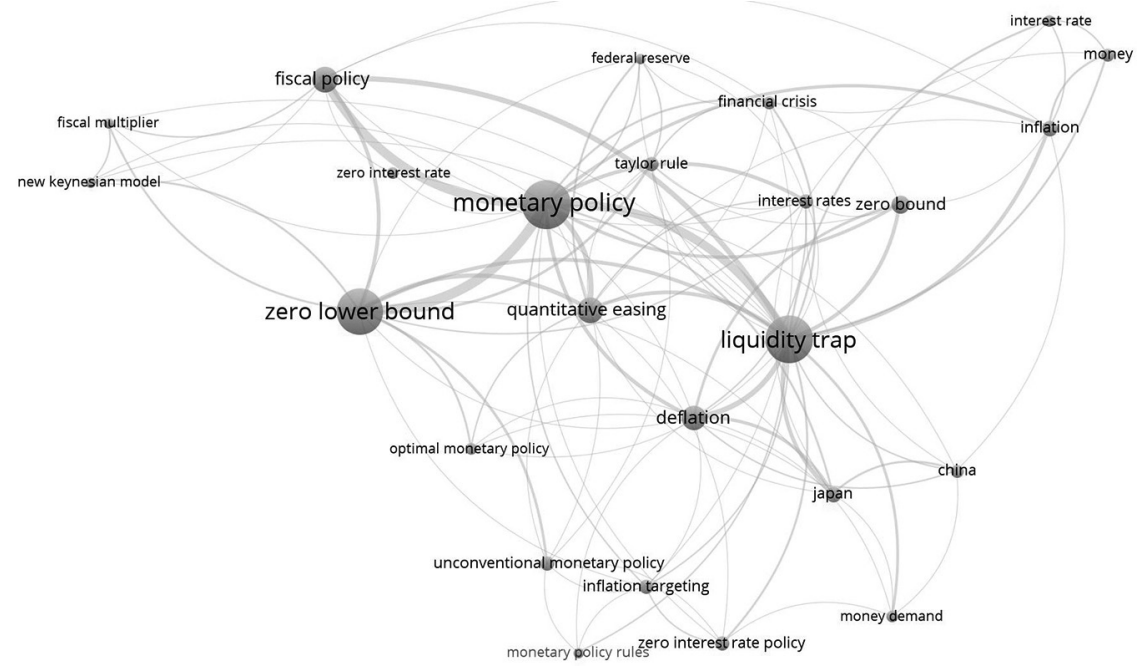

Fuente: elaboración propia con VOSviewer.

número de citas es considerado un indicador del impacto y la difusión que las ideas contenidas en una publicación tienen dentro de la comunidad científica.

La versión moderna de la trampa de liquidez, inscrita en el contexto de los modelos neokeynesianos (New Keynesian Models), difiere en aspectos fundamentales de la keynesiana tradicional. Para facilitar nuestra exposición hemos decidido agrupar el análisis de palabras clave en dos grandes temas: política monetaria y política fiscal.

\subsubsection{Política monetaria}

Uno de los aspectos que más destaca en la literatura reciente es que la política monetaria puede seguir siendo efectiva aún cuando la tasa de interés nominal de corto plazo haya alcanzado su límite inferior cero (zero lower bound, zero bound, o zero interest rate).

En la actualidad, muchos bancos centrales en el mundo conducen su política estableciendo un objetivo para la tasa de interés nominal overnight en el mercado interbancario y ajustando la oferta de dinero a ese objetivo mediante operaciones de mercado abierto. Dirigiendo los tipos de interés la autoridad monetaria gestiona las condiciones de liquidez en el mercado de dinero y trata de alcanzar la estabilidad de precios. Pero cuando la tasa de interés nominal 
ha llegado a cero o se encuentra cerca de dicho valor, el Banco Central pierde su poder para impulsar la demanda agregada. Esto se debe a que los prestamistas en general no aceptan una tasa de interés nominal negativa cuando es posible en su lugar mantener dinero en efectivo. En este escenario, se dice que la política monetaria convencional es inefectiva y el estímulo adicional a la economía deberá ser proporcionado mediante la implementación de medidas no convencionales (unconventional monetary policy). Estas medidas se pueden definir como "aquellas políticas destinadas a afectar directamente el costo y la disponibilidad de financiamiento externo para los bancos, los hogares y las empresas no financieras" (Smaghi, 2009, p. 4). En la práctica, una forma en que un Banco Central puede incidir sobre el costo del crédito es influir en las tasas de interés real de largo plazo generando expectativas de inflación. Si la inflación esperada aumenta, la tasa de interés real se reduce aún cuando la tasa de interés nominal permanezca en cero.

\subsubsection{Expectativas inflacionarias}

Krugman (1998) argumenta que la recesión japonesa de la década de los noventa puede analizarse como un caso de trampa de liquidez. En esencia, a partir de un modelo no estocástico de equilibrio general intertemporal, dicho autor plantea que la salida a este problema tiene que ver con la credibilidad que los agentes tengan con respecto a la política implementada por el Banco Central. La persistencia de la trampa de liquidez se explica por la incapacidad de la autoridad monetaria de generar las expectativas de inflación que el sistema necesita para inducir el gasto de los agentes en el periodo corriente. Eggertsson \& Krugman (2012) también argumentan en favor de la creación de expectativas inflacionarias como una de las principales salidas al problema de la trampa de liquidez.

Las implicaciones para la inestabilidad agregada del sistema económico derivadas de tomar en cuenta la presencia del límite inferior de la tasa de interés nominal, son discutidas por Benhabib, Schmitt-Grohe \& Uribe (2001) a partir de un modelo donde la política monetaria es conducida de acuerdo con una regla de Taylor (Taylor rule). La lógica de este tipo de reglas de política monetaria implica que, si la estabilidad de precios es el objetivo prioritario de las autoridades monetarias, cuando las presiones deflacionarias cedan y la inflación comience a ubicarse por encima de su nivel objetivo, el Banco Central responderá elevando la tasa de interés nominal y revirtiendo cualquier incremento previo en la base monetaria. En consecuencia, bajo este esquema la salida de la trampa de liquidez se puede ver frustrada. Nuevamente, se dice 
que la economía se encuentra atrapada en una verdadera trampa de liquidez sólo si la autoridad monetaria fracasa en su intento de modificar las expectativas del público.

Sin embargo, aunque diversos autores plantean la posibilidad teórica de un papel para la política monetaria en una trampa de liquidez, Svensson (2003) reconoce que en la práctica es complicado para un Banco Central aplicar la solución al problema. Dicho autor analiza diversas propuestas halladas en la literatura y propone lo que denomina la "forma infalible" (foolproof way) para superar la trampa de liquidez. Su propuesta (que se enfoca principalmente al caso de Japón) es una combinación de tres elementos: 1) El compromiso por parte del Banco Central para alcanzar un mayor nivel de precios en el futuro, de preferencia mediante el establecimiento de una trayectoria objetivo para el nivel de precios. 2) Una acción concreta que demuestre el compromiso del Banco Central para lograr el mayor nivel de precios futuro, que induzca las expectativas de inflación en el sector privado y reduzca la tasa de interés real. Esta acción implica una depreciación inicial del tipo de cambio (medido en unidades de moneda nacional por unidad de moneda extranjera) y una flotación controlada. 3) Una estrategia de salida que especifique cuándo y cómo regresar a la normalidad. De acuerdo con Svensson esta estrategia consiste en renunciar al control del tipo de cambio en favor de una política de metas de inflación (inflation targeting) o del nivel de precios cuando el objetivo del nivel de precios haya sido alcanzado.

\subsubsection{Deflación}

La deflación es una complicación que puede resultar cuando la economía cae en una trampa de liquidez y permanece en recesión por algún tiempo. Cuando los agentes tienen expectativas deflacionarias, la tasa de interés real (la tasa de interés nominal menos la inflación esperada) aumenta y golpea a la inversión privada al incrementar el costo del endeudamiento, lo que a su vez empeora la depresión. Benhabib et al. (2002) muestran que si un Banco Central actúa siguiendo una función de reacción del tipo regla de Taylor, las expectativas deflacionarias junto con el límite inferior cero pueden conducir a la economía a una espiral deflacionaria que la política monetaria no puede evitar. En este caso, el objetivo central del artículo es el diseño de medidas de política fiscal y monetaria que preserven la regla de Taylor (y con ella sus propiedades como mecanismo de estabilización del producto y los precios), pero que al mismo tiempo eliminen la dinámica que conduce a la economía hacia un estado estacionario de trampa de liquidez. 
Por su parte, Adam \& Billi $(2007,2006)$ discuten las implicaciones de tomar en cuenta la presencia del límite inferior para la tasa de interés nominal en un modelo neokeynesiano, en presencia de un compromiso de política monetaria y cuando ésta es conducida discrecionalmente. De acuerdo con estos autores, una calibración para la economía estadounidense sugiere que los valores bajos de la tasa de interés natural ${ }^{20}$ llevan a pérdidas considerables en el producto y a una deflación cuando la política monetaria es conducida de manera discrecional. La caída en el producto y la deflación son mayores que cuando existe un compromiso en la política monetaria y no se presentan en el modelo si se hace abstracción de la existencia de un límite inferior para la tasa de interés.

\subsubsection{Otras medidas no convencionales}

Siguiendo con el análisis de medidas de política monetaria no convencional, Cúrdia \& Woodford (2011) analizan dos alternativas que no se encuentran en el modelo neokeynesiano básico: las variaciones en el tamaño y en la composición de la hoja de balance del Banco Central; las cuales se identifican con la implementación de una política de oferta de reservas y una política de crédito del Banco Central. En términos generales, las extensiones al modelo permiten asignar un papel para la intermediación financiera. Uno de los resultados centrales es que la incorporación explícita de la hoja de balance del Banco Central no implica necesariamente que haya lugar para una política de relajamiento cuantitativo como una herramienta adicional de estabilización, aún cuando la economía se encuentre en una trampa de liquidez. Los autores invocan la experiencia del в entre 2001 у 2006 (donde la implementación del relajamiento cuantitativo fue insuficiente para impulsar la reactivación económica), para argumentar que su resultado teórico puede ser muy cercano a la realidad. En cambio, el modelo indica que puede existir un papel para la política de crédito del Banco Central (o para una compra de activos específicos) cuando los mercados financieros están dañados.

La intervención directa de la autoridad monetaria como intermediario en el mercado de crédito también es analizada por Gertler y Karadi (2011). Estos

En los modelos neokeynesianos, la tasa de interés natural se define como "la tasa de interés real de equilibrio en una economía sin fricciones monetarias" (Amato, 2005, p. 730), es decir, con precios y salarios flexibles. La presencia de una tasa natural negativa es un factor importante que en la literatura actual puede conducir a una trampa de liquidez (por ejemplo, en Krugman, 1998 y Adam \& Billi, 2006). 
autores desarrollan un modelo macroeconómico cuantitativo para analizar, en un escenario de crisis financiera simulada, los efectos de implementar una política no convencional: el Banco Central capta fondos de los hogares para canalizarlos a las empresas con el fin de compensar una ruptura en el flujo de crédito otorgado por los intermediarios financieros privados. Uno de los resultados centrales del modelo es que cuando se incorpora la presencia del límite inferior para la tasa de interés nominal y el Banco Central implementa su política de crédito en la crisis, la contracción del producto es menor que cuando no se toma en cuenta dicho límite.

Otro ejercicio interesante en el modelo de Gertler \& Karadi es la evaluación de las ganancias netas, en términos de bienestar, de la política monetaria no convencional. El modelo sugiere que, mientras los costos en términos de eficiencia son pequeños, las ganancias son muy significativas. Este resultado conduce a un análisis normativo de la política de crédito del Banco Central que resalta las bondades de su intervención directa en el mercado de crédito versus una política alternativa de inyección de capital a los intermediarios financieros privados.

Por otra parte, Hamilton \& Wu (2012) examinan opciones alternativas de política monetaria en presencia de una trampa de liquidez. Asimismo, desarrollan estimaciones empíricas de los efectos de la estructura de vencimiento de la deuda del Tesoro estadounidense en poder del público sobre la estructura temporal de tasas de interés. Esta revisión los conduce a enfocarse en una estrategia particular que consiste en tratar de influir sobre la estructura temporal de tasas de interés a través de la estructura de vencimiento de los títulos adquiridos por la FED, mediante operaciones de mercado abierto consideradas como "no estándar".

\subsubsection{Politica fiscal}

La política fiscal se sigue considerando como un instrumento importante para la salida de la trampa de liquidez, pero la razón es algo diferente a la señalada en la visión keynesiana tradicional: las medidas fiscales funcionan porque sirven como refuerzo de la política monetaria al crear mayor confianza en los agentes de que el Banco Central mantendrá su política anunciada para generar inflación y mantener la tasa de interés nominal baja, hasta que la economía salga de la depresión. Es decir, la política fiscal es un apoyo para que la política monetaria pueda incidir sobre las expectativas de los agentes. 
Por ejemplo, Benhabib et al. (2002) proponen una política de estabilización que consiste en un estímulo fiscal que se active automáticamente cuando la inflación esté disminuyendo y la economía se dirija hacia un estado estacionario de trampa de liquidez. Específicamente, la regla fiscal consiste en un superávit presupuestal sensible a la inflación, que exija una reducción de los impuestos cuando la inflación disminuya. Así, a medida que la economía se acerque a la trampa de liquidez, los déficits fiscales se volverán tan grandes que el estado estacionario con una baja inflación resultará fiscalmente insostenible, de manera que deja de ser un equilibrio compatible con el supuesto de expectativas racionales. Vale la pena señalar que, aunque los autores apelan a la política fiscal como medida para superar la trampa de liquidez, la razón es muy diferente a la que se arguye en el modelo keynesiano tradicional. Es decir, el mecanismo a través del cual actúa la expansión fiscal no es el multiplicador keynesiano, sino sus efectos sobre la restricción presupuestal intertemporal del gobierno. El segundo enfoque consiste en cambiar de una regla de tasa de interés a una regla de crecimiento de la oferta monetaria, la cual también debe estar acompañada por una política fiscal adecuada; es decir, una que a medida que la tasa de interés se acerque a cero vuelva al gobierno intertemporalmente insolvente.

\subsubsection{Multiplicador fiscal}

En los modelos neokeynesianos también hay lugar para analizar el impacto del gasto de gobierno a través del multiplicador fiscal. Woodford (2011) examina los factores que determinan el tamaño del multiplicador del gasto de gobierno bajo diferentes escenarios de política monetaria. Aunque el autor realiza experimentos de política en el contexto de diferentes modelos (neoclásico básico, neokeynesiano sin una especificación particular sobre el ajuste de precios y salarios y neokeynesiano con salarios flexibles y ajuste escalonado de precios) y formulaciones para la regla de Taylor, el caso que nos resulta de mayor interés es cuando toma en cuenta la presencia del límite inferior de la tasa de interés nominal de corto plazo. La cuestión que el autor evalúa en ese modelo es el efecto de elegir un gasto de gobierno elevado durante una crisis (la cual se manifiesta en un aumento en el diferencial de tasas de interés provocado por un disturbio en el sector financiero), tomando como dado el valor del gasto de gobierno durante los tiempos normales (es decir, cuando no hay crisis) y una política monetaria descrita por una regla de Taylor. En este modelo el multiplicador que se obtiene es mayor a $1 \mathrm{y}$, de hecho, puede ser 
muy superior a 1. Por tanto, el autor concluye que cuando la tasa de interés se encuentra restringida por su límite inferior, el gasto de gobierno puede proporcionar un impulso importante para elevar el producto y el nivel de bienestar en la economía.

Woodford enfatiza que si bien el efecto de un estímulo fiscal es muy favorable cuando la política monetaria está restringida por el límite inferior de la tasa de interés nominal, la política fiscal expansiva debe cancelarse cuando la trampa de liquidez haya sido superada. Esto se debe a que el incremento en los impuestos que se puede requerir para pagar la deuda pública una vez superada la crisis, puede reducir significativamente el efecto favorable de la expansión fiscal durante la crisis, tanto en términos de producto como de bienestar.

La magnitud del multiplicador del gasto también es evaluada por Christiano, Eichenbaum \& Rebelo (2011) en un marco neokeynesiano bajo diferentes escenarios. El caso que se abordará es el que deriva de la versión del modelo sin capital y donde la tasa de interés nominal es constante debido a que ha alcanzado su límite inferior. El multiplicador que resulta es aproximadamente tres veces mayor al multiplicador que se obtendría si la tasa de interés nominal estuviera por encima de cero. La intuición de por qué el multiplicador puede ser tan grande cuando la tasa de interés permanece constante en cero es la siguiente: el aumento en el gasto de gobierno lleva a un aumento del producto, el costo marginal y la inflación esperada. Con una tasa de interés nominal nula, el aumento en la inflación esperada lleva a la baja a la tasa de interés real, lo que genera un ascenso en el gasto privado (una reducción en el ahorro deseado). La expansión en el gasto genera un aumento adicional en el producto, el costo marginal y la inflación esperada lo que lleva a una disminución adicional en la tasa de interés real. El resultado neto es un gran aumento en la inflación y el producto.

Otra conclusión interesante con respecto al papel de la política fiscal en una trampa de liquidez es la que obtienen Eggertsson \& Krugman (2012). El modelo que desarrollan estos autores muestra que contrario a lo que se piensa generalmente, una depresión causada por un sobreendeudamiento del sector privado puede ser solucionada incurriendo en más deuda, pero en este caso deuda del sector público. Es decir, un gasto de gobierno financiado con déficit puede ayudar a que la economía evite el desempleo y la deflación en tanto los agentes privados endeudados logran sanear sus hojas de balance. 


\section{CONCLUSIONES}

Sin duda, la depresión japonesa de los años noventa y la crisis financiera global de 2007-2008 fueron los eventos clave que renovaron el interés académico por analizar el papel de la política monetaria en un escenario de bajas tasas de interés. Asimismo, el marco teórico neokeynesiano en el que se inscribe la mayoría de estos estudios ha dado lugar a nuevas conclusiones que no se encuentran en el viejo enfoque. Por ejemplo, la política monetaria no es totalmente ineficaz en un escenario de trampa de liquidez. Las medidas no convencionales aparecen como una herramienta alternativa del Banco Central para incidir sobre la demanda agregada y el nivel de precios, aunque reducir las tasas de interés a cero ha demostrado ser insuficiente para reactivar la actividad y se presenta el riesgo de deflación en varios países ricos. "Aun cuando muchos políticos siguen comprometidos con la consolidación fiscal, muchos economistas ahora argumentan que el estímulo fiscal insuficiente ha sido uno de los mayores fracasos de la era posterior a la crisis". ${ }^{21}$

Por lo tanto, la política fiscal sigue considerándose un elemento relevante, porque además del mecanismo del multiplicador, puede actuar como un refuerzo de la política monetaria en la generación de expectativas inflacionarias. En consecuencia, se debe diseñar una estrategia coordinada de política económica para impulsar el crecimiento económico sostenible y reducir los niveles de deuda y déficit. Para lograr lo anterior, se requieren reformas estructurales para restaurar la sostenibilidad fiscal, por ejemplo, una reforma fiscal que realmente contribuya a sanear las finanzas públicas y se asigne el gasto de manera eficiente.

Finalmente, la información obtenida a partir del análisis bibliométrico ha sido de gran utilidad para develar las tendencias actuales de investigación asociadas al tema de la trampa de liquidez. 


\section{ANEXO}

Anexo 1. Los 12 artículos más citados sobre la trampa de liquidez

\begin{tabular}{|c|c|c|c|}
\hline Autor(es) & Título & Año & Citas \\
\hline Krugman, P.R. & $\begin{array}{l}\text { It's baaack: Japan's Slump and The Return of the } \\
\text { Liquidity Trap. }\end{array}$ & 1998 & 354 \\
\hline $\begin{array}{l}\text { Christiano, L., Eichenbaum, M. } \\
\text { \& Rebelo, S. }\end{array}$ & When is The Government spending Multiplier Large? & 2011 & 208 \\
\hline Gertler, M. \& Karadi, P. & A Model of Unconventional Monetary Policy. & 2011 & 205 \\
\hline $\begin{array}{l}\text { Benhabib, J., Schmitt-Grohé, S. } \\
\text { \& Uribe, M. }\end{array}$ & The Perils of Taylor Rules. & 2001 & 168 \\
\hline Eggertsson, G.B. \& Krugman, P. & $\begin{array}{l}\text { Debt, deleveraging, and The Liquidity Trap: A Fisher- } \\
\text { Minsky-Koo Approach. }\end{array}$ & 2012 & 138 \\
\hline Woodford, M. & $\begin{array}{l}\text { Simple Analytics of The Government Expenditure } \\
\text { Multiplier. }\end{array}$ & 2011 & 134 \\
\hline Svensson, L.E.0. & $\begin{array}{l}\text { Escaping from A Liquidity Trap and Deflation: The } \\
\text { Foolproof Way and Others. }\end{array}$ & 2003 & 109 \\
\hline $\begin{array}{l}\text { Benhabib J., Schmitt-Grohé S. } \\
\text { \& Uribe M. }\end{array}$ & Avoiding Liquidity Traps. & 2002 & 94 \\
\hline Hamilton, J.D. \& Wu, J.C. & $\begin{array}{l}\text { The Effectiveness of Alternative Monetary Policy } \\
\text { Tools in a Zero Lower Bound Environment. }\end{array}$ & 2012 & 74 \\
\hline Adam, K. \& Billi, R.M. & $\begin{array}{l}\text { Optimal Monetary Policy under Commitment with A } \\
\text { Zero Bound on Nominal Interest Rates. }\end{array}$ & 2006 & 68 \\
\hline Orphanides, A. \& Wieland, V. & Efficient Monetary Policy Design near Price Stability. & 2000 & 65 \\
\hline Cúrdia, V. \& Woodford, M. & $\begin{array}{l}\text { The Central-bank Balance Sheet As An Instrument of } \\
\text { Monetary Policy. }\end{array}$ & 2011 & 57 \\
\hline
\end{tabular}

Fuente: elaboración propia con información de Scopus (fecha de actualización: 19 de octubre de 2016). 


\section{BIBLIOGRAFÍA}

Adam, K. \& Billi, R. M. (2006), "Optimal Monetary Policy under Commitment with a Zero Bound on Nominal Interest Rates", Journal of Money, Credit and Banking, 38(7), 1877-1905.

\& Billi, R. M. (2007), "Discretionary Monetary Policy and The Zero Lower Bound on Nominal Interest Rates", Journal of Monetary Economics, 54(3), 728-752.

Amato, J. D. (2005), "The Role of the Natural Rate of Interest in Monetary Policy", CESifo Economic Studies, 51(4), 729-755.

Ashworth, J. (2013), "Quantitative easing by The Major Western Central Banks during The Global Financial Crisis", en Steven N. Durlauf y Lawrence E. Blume (eds.), The New Palgrave Dictionary of Economics Online, Palgrave Macmillan. Recuperado el 1 de octubre de 2015.

Benhabib, J., Schmitt-Grohe, S. \& Uribe, M. (2001), "The Perils of Taylor Rules", Journal of Economic Theory, vol. 96, 40-69.

(2002), "Avoiding Liquidity Traps", Journal of Political Economy, vol. 110 (3), 535-563.

Bernanke, B. S. (2009), The Crisis and the Policy Response. Discurso presentado en la Stamp Lecture, London School of Economics, Londres, Inglaterra, enero 13.

Boianovsky, M. (2003), "The IS-LM model and the Liquidity Trap Concept: from Hicks to Krugman", Serie de textos para discussao (182), 1-49.

Christiano, L., Eichenbaum, M., \& Rebelo, S. (2011), "When is The Government spending Multiplier Large?”, Journal of Political Economy, 119(1), $78-121$.

Chun-Hao, C. \& Jian-Min, Y. (2012), "A Bibliometric Study of Financial Risk Literature: a Historic Approach”, Applied Economics, vol. 44(22), 2827-2839.

Clarida, R., Galí, J. \& Gertler, M. (1999), "The Science of Monetary Policy: a New Keynesian Perspective", Journal of Economic Literature, vol. XxXvII, 1661-1707, diciembre.

Cúrdia, V. \& Woodford, M. (2011), "The Central-bank Balance Sheet As An Instrument of Monetary Policy”, Journal of Monetary Economics, 58(1), 54-79.

Eggertsson, G. B. \& Krugman, P. (2012), "Debt, deleveraging, and The Liquidity Trap: A Fisher-Minsky-Koo approach", The Quarterly Journal of Economics, vol. 127(3), 1469-1513. 
Fawley, B. W. \& Neely, C. J. (2013), "Four Stories of Quantitative easing", Federal Reserve Bank of St. Louis Review, vol. 95(1), 51-88.

Gertler, M. \& Karadi, P. (2011), "A Model of Unconventional Monetary Policy”, Journal of Monetary Economics, vol. 58, 17-34.

Goodfriend, M. \& King, R. G. (1997), "The New Neoclassical Synthesis and The Role of Monetary Policy”, NBER Macroeconomics Annual, vol. 12, 231-296.

Hamilton, J. D. \& Wu, J.C. (2012), "The Effectiveness of Alternative Monetary Policy Tools in A Zero Lower Bound Environment", Journal of Money, Credit and Banking, 44 (SUPPL. 1), 3-46.

Hicks, J. R. (1937), "Mr. Keynes and The "Classics": A Suggested Interpretation”, Econometrica, vol. 5, 147-59, abril.

(1939)[1976], Valor y capital. Investigación sobre algunos principios fundamentales de teoría económica, 2a ed., México, Fondo de Cultura Económica.

Hsu, C. L., \& Chiang, C. H. (2015), "The Financial Crisis Research: A Bibliometric Analysis", Scientometrics, vol. 105(1), 161-177.

Keynes, J. M, (1936) [2006], Teoría general de la ocupación el interés y el dinero, $4^{\text {a }}$. ed., México, Fondo de Cultura Económica.

Krugman, P. (1998), "It's baaack! Japan's Slump and The Return of the Liquidity Trap", Brookings Papers on Economic Activity, (2), 137-205.

Obregón, C. (2011), La crisis financiera mundial. Perspectivas de México y de América Latina, México, Siglo XXI Editores.

Orphanides, A. \& Wieland, V. (2000), "Efficient Monetary Policy Design near Price Stability", Journal of The Japanese and International Economies, vol. 14 (4), 327-365.

Prado, J. W., Castro Alcántara, V., Melo Carvalho, F., Vieira, K.C., Machado, L.K.C. \& Tonelli, D.F. (2016), "Multivariate Analysis of Credit Risk and Bankruptcy Research Data: A Bibliometric Study involving Different knowledge Fields (1968-2014)", Scientometrics, vol. 106 (3), 1007-1029.

Robertson, D. H. (1940), "Mr. Keynes and the Rate of Interest", Essays in Monetary Theory, London, P. S. King \& Son, Ltd.

Smaghi, L. B. (2009), "Conventional and Unconventional Monetary Policy", Keynote Lecture at the International Centre for Monetary and Banking Studies (Ісмв), Genova, 1-21.

Snowdon, B. \& Vane, H.R. (2005), Modern Macroeconomics. It's Origins, Development and Current State, Reino Unido, Edward Elgar; Cheltenham, 807. 
Eva Ugarte, Josefina León y Gilberto Parra

Svensson, L. E. O. (2003), "Escaping from a Liquidity Trap and Deflation: The Foolproof Way and Others", Journal of Economic Perspectives, vol. $17(4), 145-166$.

Van Eck, N. J., Waltman, L., Dekker, R. \& Van Den Berg, J. (2010), "A Comparison of Two Techniques for Bibliometric Mapping: Multidimensional Scaling and vos", Journal of the American Society for Information Science and Technology, vol. 61(12), 2405-2416.

Van Eck, N. J. \& Waltman, L. (2010), "Software Survey: vosviewer, A Computer Program for Bibliometric Mapping”, Scientometrics, vol. 84(2), 523538.

(2011), "Text Mining and Visualization using vosviewer", ISSI Newsletter, vol. 7 (3), 50-54.

(2014), "Visualizing Bibliometric Networks", en Y. Ding, R. Rousseau \& D. Wolfram (eds.), Measuring Scholarly Impact: Methods and Practice, Springer.

Woodford, M. (2003), Interest and Prices. Foundations of a Theory of Monetary Policy, Estados Unidos, Princeton University Press, New Jersey, 785.

(2011), "Simple Analytics of the Government Expenditure Multiplier", American Economic Journal, vol. 3, 1-35, enero. 